ISSN 1112-9867

Available online at

http://www.jfas.info

\title{
ARTIFICIAL INTELLIGENCE TO PREDICT INHIBITION PERFORMANCE OF PITTING CORROSION
}

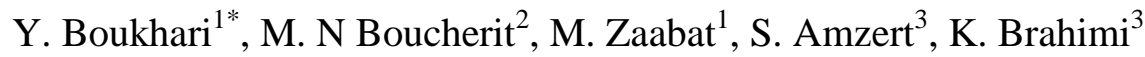 \\ ${ }^{1}$ Oum El Bouaghi University, Oum El Bouaghi 04000, Algeria \\ ${ }^{2}$ Research \& Development Unit in Nuclear Engineering - URDIN, Algiers - Algeria \\ ${ }^{3}$ Nuclear Research Center of Birine - Algeria
}

Received: 26 June 2016 / Accepted: 22 December 2016 / Published online: 01 January 2017

\begin{abstract}
This work aims to compare several algorithms for predicting the inhibition performance of localized corrosion. For this more than 400 electrochemical experiments were carried out in a corrosive solution containing an inorganic inhibitor. Pitting potential is used to indicate the performance of the inhibitor/oxidant mixture to prevent pitting corrosion.

At the end of the electrochemical program a file containing all the experimental results has been prepared and submitted to several algorithms. Through a training phase each algorithm uses a set of experimental results to adjust its parameters and another set to predict the pitting potential starting from the properties and the chemical composition of the solution. The prediction performance of an algorithm is estimated by the difference between experimental pitting potential and the calculated one. The order of performance of the algorithms is: GA-ANN > LS-SVM > PSO-ANN $>$ ANN $>$ ANFIS $>$ KNN $>$ RT $>$ KBP $>$ LDA.
\end{abstract}

Key words: Pitting potential, Corrosion inhibitor, Performance prediction, Artificial intelligence.

Author Correspondence, e-mail: jacoubchimie@yahoo.fr

doi: http://dx.doi.org/10.4314/jfas.v9i1.19 


\section{INTRODUCTION}

Any industrial installation with solid/liquid interfaces is exposed to corrosion risks. If some forms of corrosion can be estimated and controlled, others remain unpredictable and pernicious. Pitting corrosion is one of the major industrial threats despite all the studies that have been devoted to it $[1,2]$. In aqueous medium and during a metastable phase, pits appearing on metal surfaces are microscopic and undetectable [3]. When pits become stable, they evolve so quickly that they can cause unexpected and irreversible material damages. Fighting such a threat can only be preventive for example by the use of chemical inhibitors. For a secondary cooling circuit of a nuclear installation, made of carbon steel and open to the atmosphere, the localized corrosion inhibitors should also be chemically stable and non-toxic in order to avoid any damage to the environment after the periodic water draining [4].

In the present work we have been interested by inorganic inhibitors: tungstate, molybdate whose efficiency has been described in a previous study [5], and also silicates, carbonates, bicarbonates and three forms of phosphates.

Many studies were conducted in order to understand the chemical process specific to each inhibitor [6,7]. But what is observed is that in the majority of studies, the performance is interpreted in terms of the variation of local $\mathrm{pH}$, ionic conductivity or passive film properties. The inhibition performance has rarely been attributed to the specific chemistry of the inhibitor. A consensual fact for all studies is the use of the pitting potential $\mathrm{E}_{\mathrm{pit}}$ as an inhibition performance indicator. This parameter is usually taken from voltammograms obtained from linear potential sweep voltammetry. If experimentally an anodic shifting of this parameter indicates an inhibition performance, theoretically it is extremely difficult to predict the value of this parameter on the basis of the chemical composition of the medium. Under these conditions, the artificial intelligence through the use of training algorithms can be a feasible and effective alternative.

Among modern algorithms LS-SVM, KNN, LDA, ANN, PSO-ANN, ANFIS, GA-ANN, RT and KBP are based on training and testing steps.

Least Squares Support Vector Machine (LS-SVM) is an algorithm recommended to solve both discrimination and prediction problems through solving a set of linear equations. The technique has been proposed by Suykens and Vandewalle [8]. Its principle is based on delimitation. It has been applied with some enthusiasm in various fields especially in the prediction of corrosion stress [9] or pits prediction [10]. K-Nearest Neighbors (KNN) algorithm is a discrimination and 
prediction tool. Its principle is to take into account at the same time the $\mathrm{K}$ samples destined to training whose variables are the closest to those of the new entry $\mathrm{x}$, by a distance to be defined. The technique has been applied recently in some studies relating to localized corrosion [11]. Linear Discriminant Analysis (LDA) is however an older method. Its principle is to find a linear combination of parameters. An example for the application of this algorithm is the study for modeling offshore pipeline failures [12]. Artificial Neural Networks (ANN) is among the largely used prevision algorithms. The principle is based on the adjustment during the training phase of multiplication parameters. In a previous study, we concluded about the performance of the algorithm for pitting potential prediction [5]. Particle Swarm Optimization (PSO) is a metaheuristic procedure invented by Russell Eberhart [13]. The algorithm is inspired by the movement of a group of birds [14]. Its principle is based on a set of potential solutions evolving to approach a convenient solution. The aim is to find the global optimum. At each iteration, the movement of each solution considers the previous position, the environment and the previous environment position. PSO-ANN is a combination of PSO and ANN that allows to get the best out of these two powerful algorithms [15].

Adaptive neuro-fuzzy inference system: (ANFIS) is an artificial neural network algorithm type that is based on Takagi-Sugeno fuzzy inference system. Its inference system corresponds to a set of fuzzy if-then rules that have training capability to approximate nonlinear functions. A recent study compared the application of ANFIS with ANN to estimate the corrosion rate of nanoceramic layers of zirconium [16]. Genetic Algorithm GA has been popularized by John Holland [17]. The principle is based on finding the extrema of a function defined on a data space. The combined GA-ANN algorithm has a great potential to handle problems such as optimization in complicated nonlinear systems [18], it can determine the best parameters for the ANN.

Also In a recent study on concrete reinforcement data predictions were made by combining ANN, ANFIS and GA [19]. Classification Tree (DT) and Regression Tree (RT) method has been developed by Leo Breiman [20] for classification or regression purpose depending on the response variable which is either categorical or numerical. A recent study mixing various algorithms among them classification tree has been conducted for pitting corrosion detection [10]. Kernel Basis Pursuit (KBP) algorithms introduced by V. Guigue, A. Rakotomamonjy, S. Canu [21] is also a fast parameter-free method that allows to estimate non-uniform-sampled functions, it gives good results on artificial and real data. 


\section{EXPERIMENTAL}

All the electrochemical investigations were carried out on a carbon steel whose chemical composition is given in Table 1 . The analysis has been made by $\mathrm{X}$-ray fluorescence technique (XRF).

Table 1: Chemical composition of the steel

\begin{tabular}{ccccccccc}
\hline Elements & $\mathrm{C}$ & $\mathrm{Mn}$ & $\mathrm{Si}$ & $\mathrm{P}$ & $\mathrm{Ti}$ & $\mathrm{Ni}$ & $\mathrm{Cu}$ & $\mathrm{Mo}$ \\
\hline$\%$ mass & 0,18 & 0,52 & 0,22 & 0,009 & 0,003 & 0,039 & 0,63 & 0,015 \\
\hline
\end{tabular}

In the experimental program, we planned 419 electrochemical experiments. With each experience we prepare a solution containing some amount of $\mathrm{NaCl}$. Chloride plays the role of the pitting corrosion initiator. We add another quantity of one of the inhibitors: $\mathrm{Na}_{2} \mathrm{WO}_{4}, \mathrm{Na}_{2} \mathrm{MoO}_{4}$, $\mathrm{NaHCO}_{3}, \mathrm{Na}_{2} \mathrm{CO}_{3}, \mathrm{Na}_{3} \mathrm{PO}_{4}, \mathrm{Na}_{2} \mathrm{HPO}_{4}, \mathrm{NaH}_{2} \mathrm{PO}_{4}$ or $\mathrm{SiO}_{3}$. Then we complete by an amount of the oxidizing agent $\mathrm{KIO}_{3}$. As we have shown in a previous paper [5], the oxidant should enhance the efficiency of the inhibitor.

Once the solution prepared, we note its $\mathrm{pH}$ and its ionic conductivity. All the electrochemical experiments were performed in an aerated cell containing three electrodes: the working electrode made of carbon steel, the auxiliary electrode made of a platinum sheet and a saturated calomel electrode (SCE) used as a reference electrode. We used a PAR273A potentiostat connected to a computer controlled by SoftCorr III software. Each experiment consists in tracing a voltammogram of the working steel in the prepared solution by sweeping its potential from -0.8 to $1 \mathrm{~V} / \mathrm{SCE}$ at a scan rate of $1 \mathrm{mV} / \mathrm{s}$.

At the end of the experiment, the pitting potential is determined from the voltammogram. It corresponds to the potential at which the current density increases sharply.

At the end of the experimental program we compiled a table of $419 \mathrm{X} 13$. Each experience is described by a row. The columns from 1 to 12 correspond respectively to ionic conductivity, $\mathrm{pH}$, $\mathrm{NaCl}, \mathrm{KIO} 3, \mathrm{Na} 3 \mathrm{PO} 4, \mathrm{Na} 2 \mathrm{HPO} 4, \mathrm{NaH} 2 \mathrm{PO} 4, \mathrm{Na} 2 \mathrm{CO} 3, \mathrm{NaHCO}, \mathrm{Na} 2 \mathrm{MoO} 4, \mathrm{Na} 2 \mathrm{WO} 4$ and $\mathrm{SiO} 3$ concentrations. In one experiment, we cannot use more than one inhibitor, and the last column (13) is reserved to the experimentally obtained pitting potential $\mathrm{E}_{\text {pit-exp. }}$ 
Mathematical processing of the experimental table was made in Matlab 8.1. Matlab possesses multiple algorithms or pre-programmed functions and it is also possible to integrate additional tools through a toolbox. All the algorithms have been executed through M-files that contain specific instructions for each algorithm.

Each algorithm considers the first 12 columns of the experimental table as input data to calculate a pitting potential $\mathrm{E}_{\mathrm{pit} \text {-cal }}$. The performance of the algorithm is then estimated by a correlation coefficient $\mathrm{R}$ which takes into consideration the difference between $\mathrm{E}_{\text {pit-cal }}$ and $\mathrm{E}_{\text {pit-exp. }}$. Before the mathematical processing, the experimental table is divided in two parts. Each algorithm exploits two third of the data table for training and the remaining part for the test.

\section{RESULTS AND DISCUSSION}

\subsection{Experimental reading of the pitting potential}

In an electrochemical study the only way to measure the performance of a pitting corrosion inhibitor remains the value of the pitting potential. However, relying on this indicator has two major problems: the difficulty in expressing this parameter from the knowledge of the physicochemical properties of the solution and also taking the exact numerical value from a voltammogram. In order to show this difficulty to get experimentally the pitting potential we superimposed on Figure 1 several voltammograms.

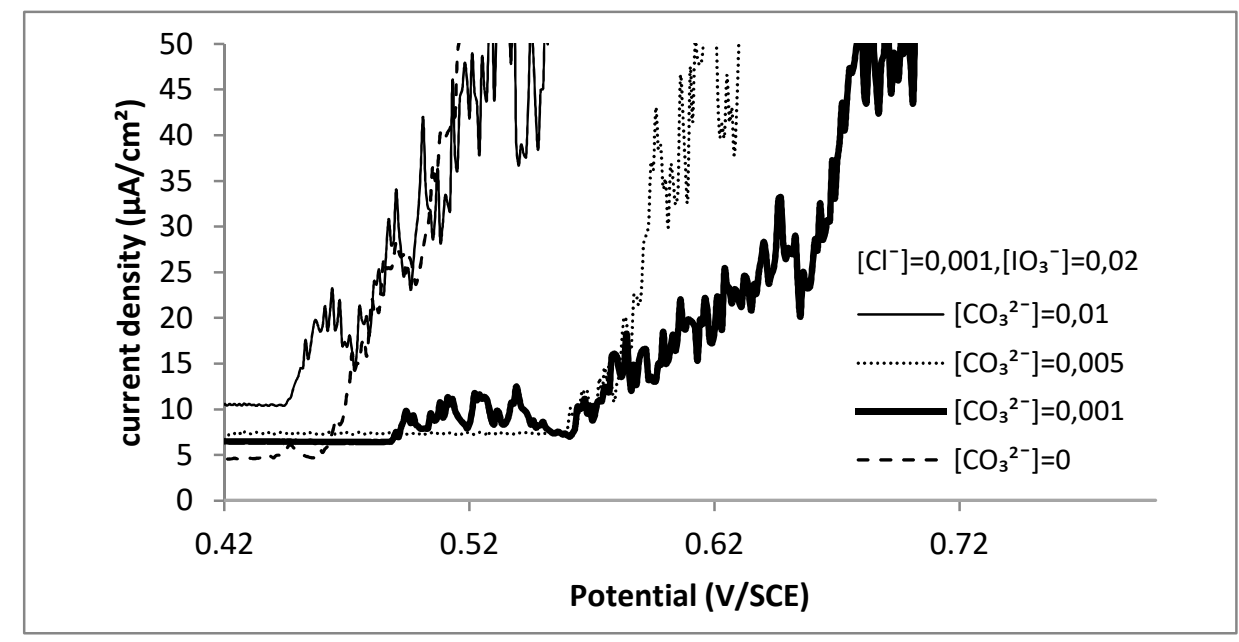

Fig.1. Various shapes of the experimental current-potential curve and the difficulty in determining the pitting potential. 
The current sensitivity is $1 \mu \mathrm{A}$. With this limitation it is difficult to observe currents due to metastable pits that are much lower. On another side pitting initiation can be followed by repassivation phenomena if the $\mathrm{pH}$ of the solution is not too low. This usually results in a lower slope of the current density through which it is difficult to decide on the exact value of the pitting potential. In the following, we consider that the pitting potential is reached when we observe a sharp increase in the current.

\subsection{Effect of $\mathrm{pH}$ on the pitting penitential:}

Many studies show that $\mathrm{pH}$ has an important role in pitting resistance [22, 23]. If we admit that the pitting process is carried out in four phases: passive film formation, passive film breakdown, metastable pits apparition, irreversible evolution of pits [24], it becomes clear that increasing the $\mathrm{pH}$ will supply hydroxides that promotes the formation of iron hydroxides which precipitate and reinforce the passive film.

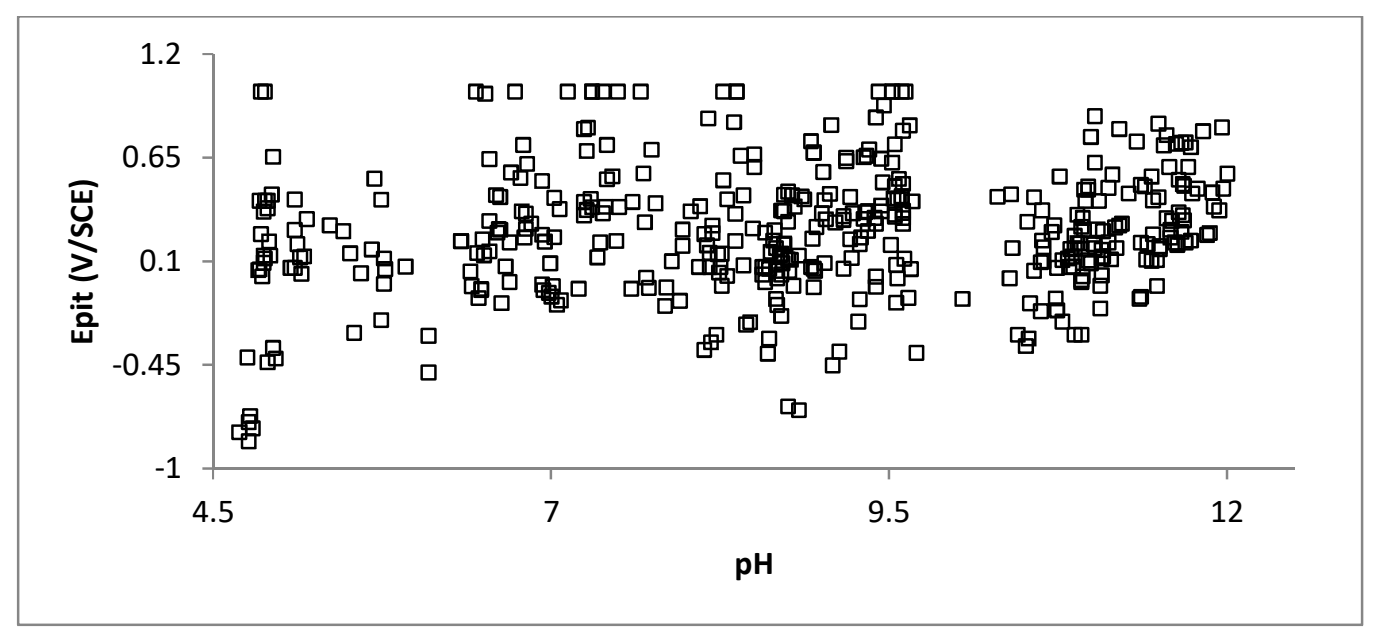

Fig.2. Effect of pH on the pitting potential.

Considering all the experiences, we tried to illustrate the evolution of the pitting potential based solely on the $\mathrm{pH}$ Figure 2. It is clear at first glance that the $\mathrm{pH}$ of the solution does not affect the pitting potential. This is because during the pitting process the oxidation of the iron produces ferrous ions which quickly form unstable species including $\mathrm{FeOH}^{+}$and $\mathrm{Fe}(\mathrm{OH})_{2}$. This reduces quickly the local $\mathrm{pH}$ making the environment of the pit very acidic while the overall $\mathrm{pH}$ of the solution remains high.

The global $\mathrm{pH}$ of the solution is then not the main parameter which determines the pitting potential value, the concentrations of chlorides inhibitors and oxidants and also the ionic 
conductivity are parameters which affect $\mathrm{E}_{\mathrm{pit}}$ value. Due to the diversity of effects of these reagents as well as the synergy they must have with the oxidant, it would be useful to have a mathematical tool that would consider all the experimental parameters in a multidimensional vector and would be able to predict the pitting potential value.

\subsection{Predictive modeling with Artificial intelligence}

In this study, we were interested in different algorithms used with varying degrees of success in the field of data mining. These algorithms are similar in the fact that they consider two phases. In the first phase called training phase a fraction of the experiments is considered. Experimental parameters are considered as input and the pitting potential is taken as the output. During this phase, the algorithm adjusts its own parameters in order to match the input data to the output. During the second phase, the parameters of the algorithm are kept constant and by considering the remaining experiments, for each vector of experimental input data the algorithm calculates an

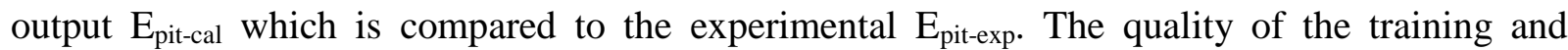
prediction are appreciated by either the correlation coefficient $\mathrm{R}$ or the normalized mean square error NMSE, which in the ideal case approach respectively 1 and 0.

In the following Figure 3, we can see the distribution of the calculated pitting potential versus the experimental pitting potential. For each algorithm, we have two figures: one corresponding to the training phase and the other to the testing phase. R and NMSE are reported on each figure.
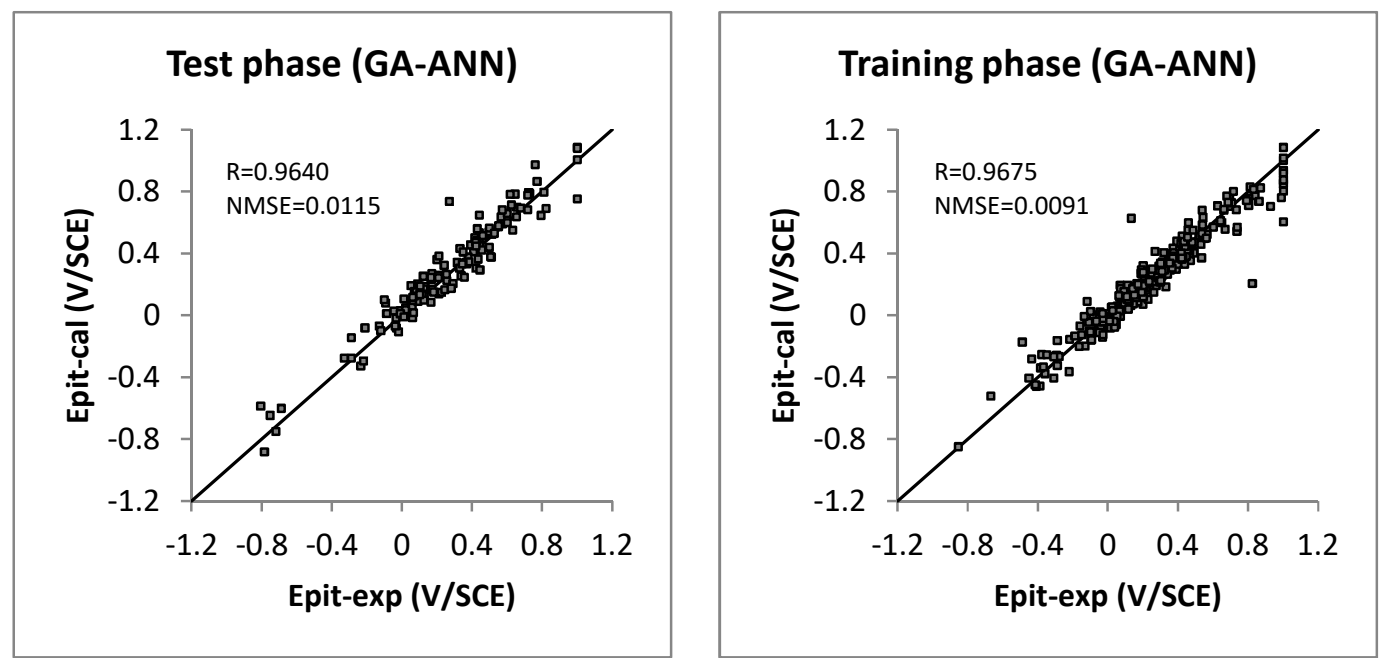

(a) 

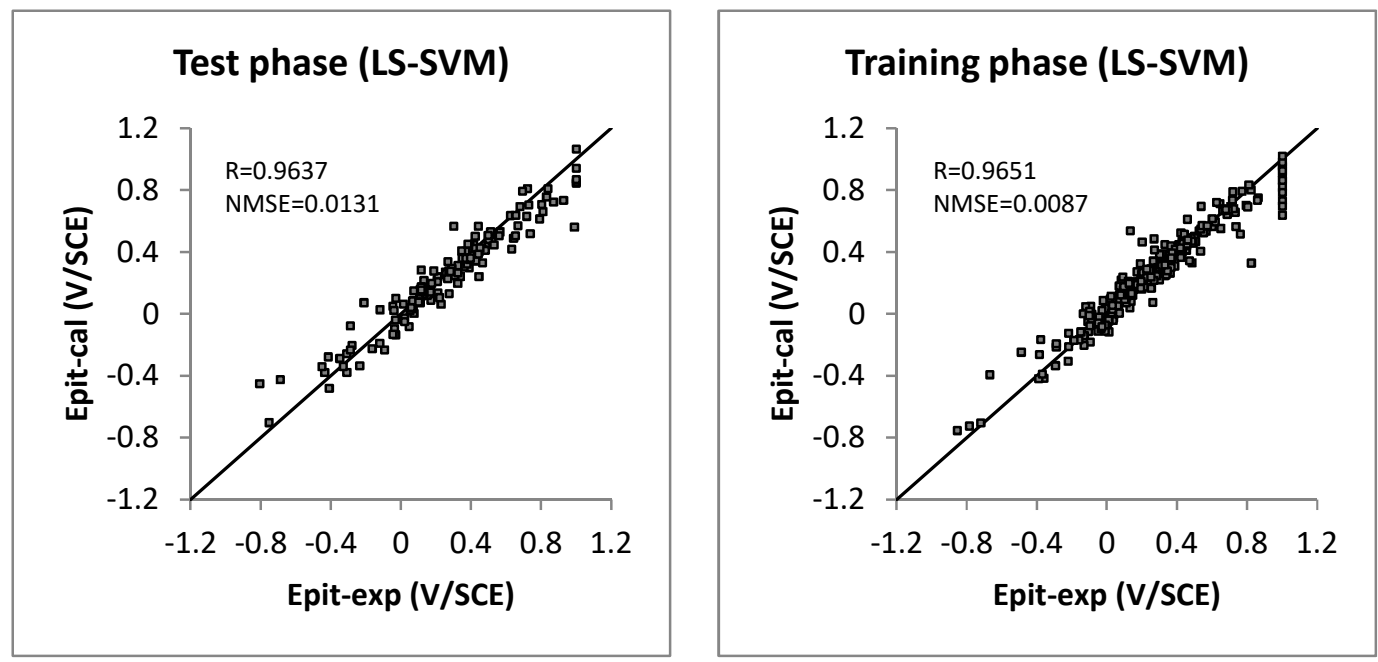

(b)
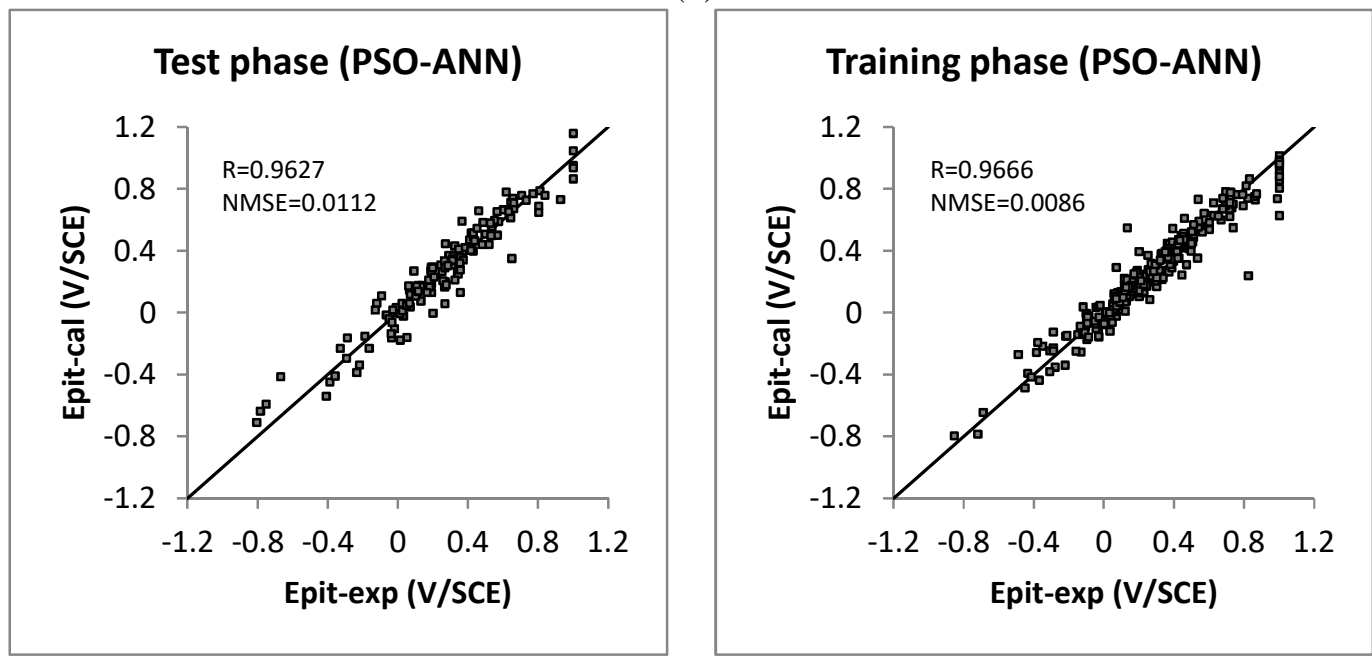

(c)
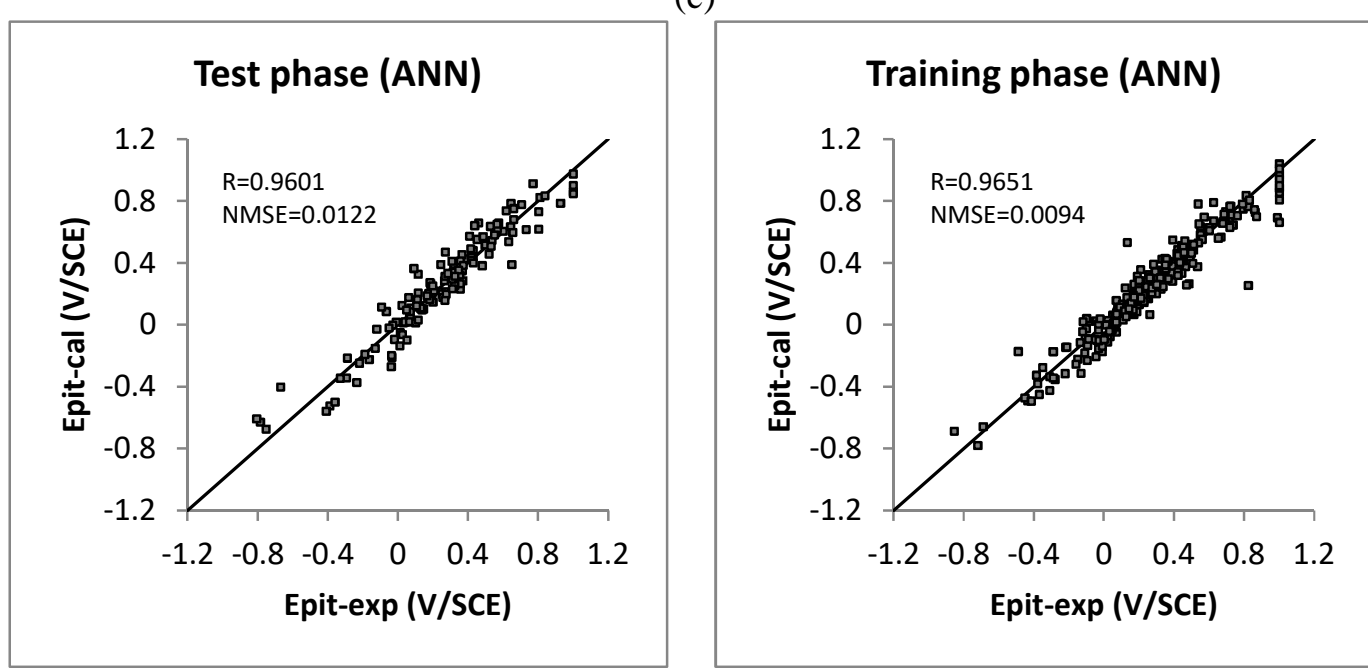

(d) 

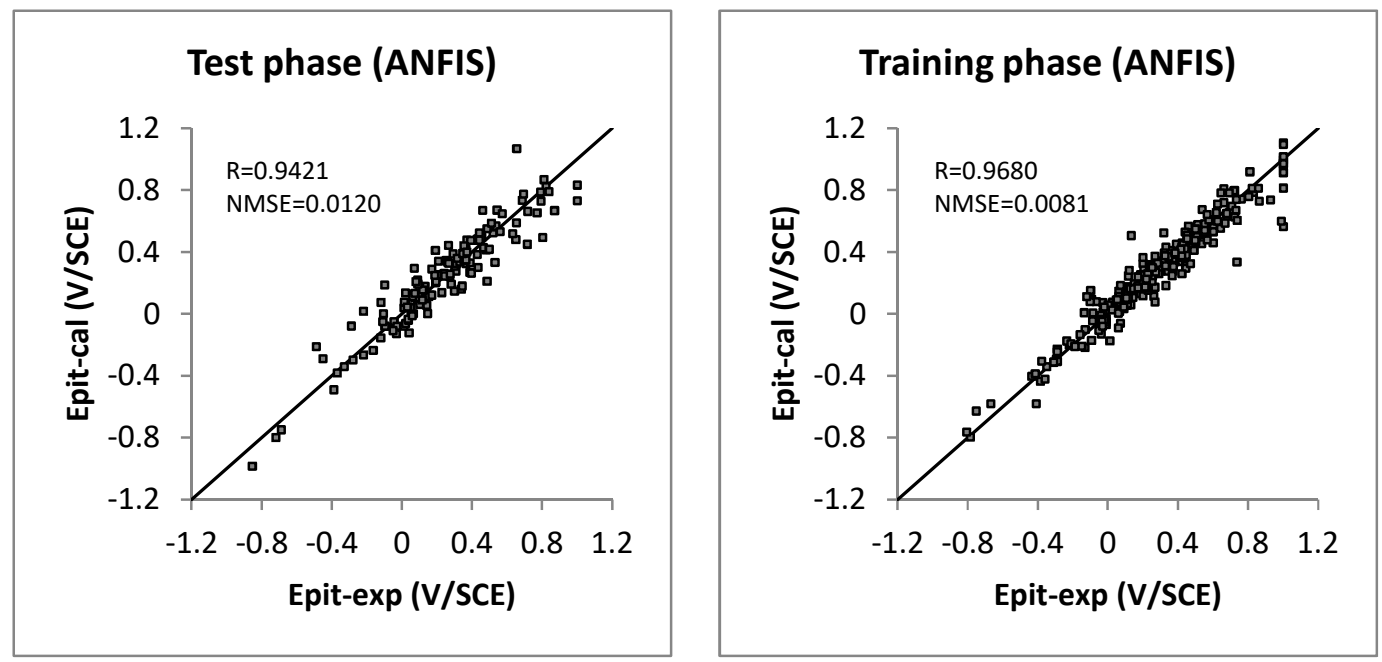

(e)
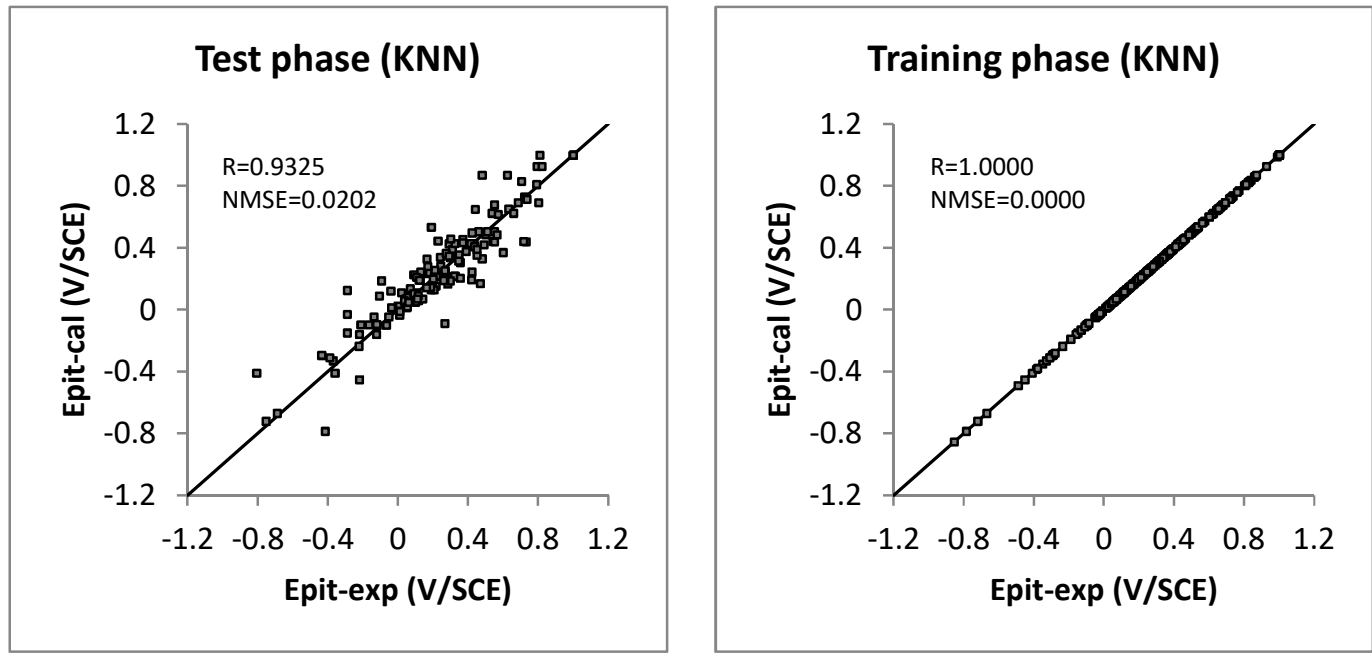

(f)
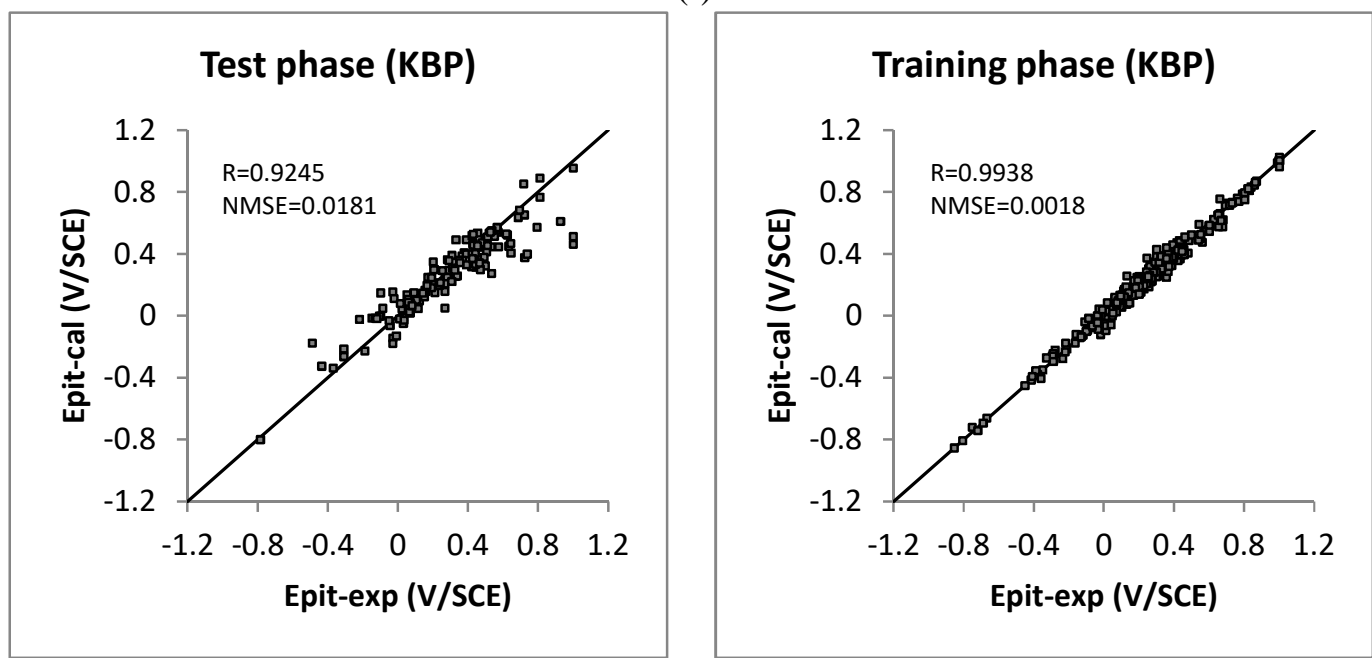

(g) 

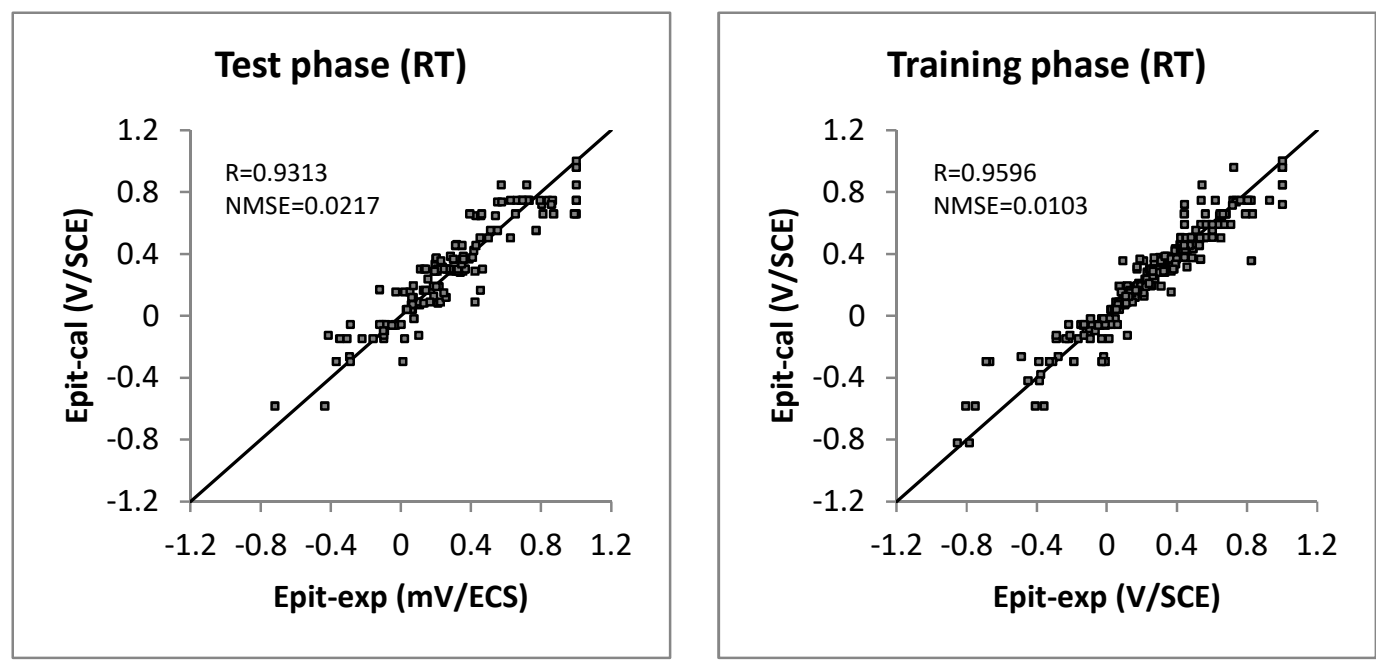

(h)
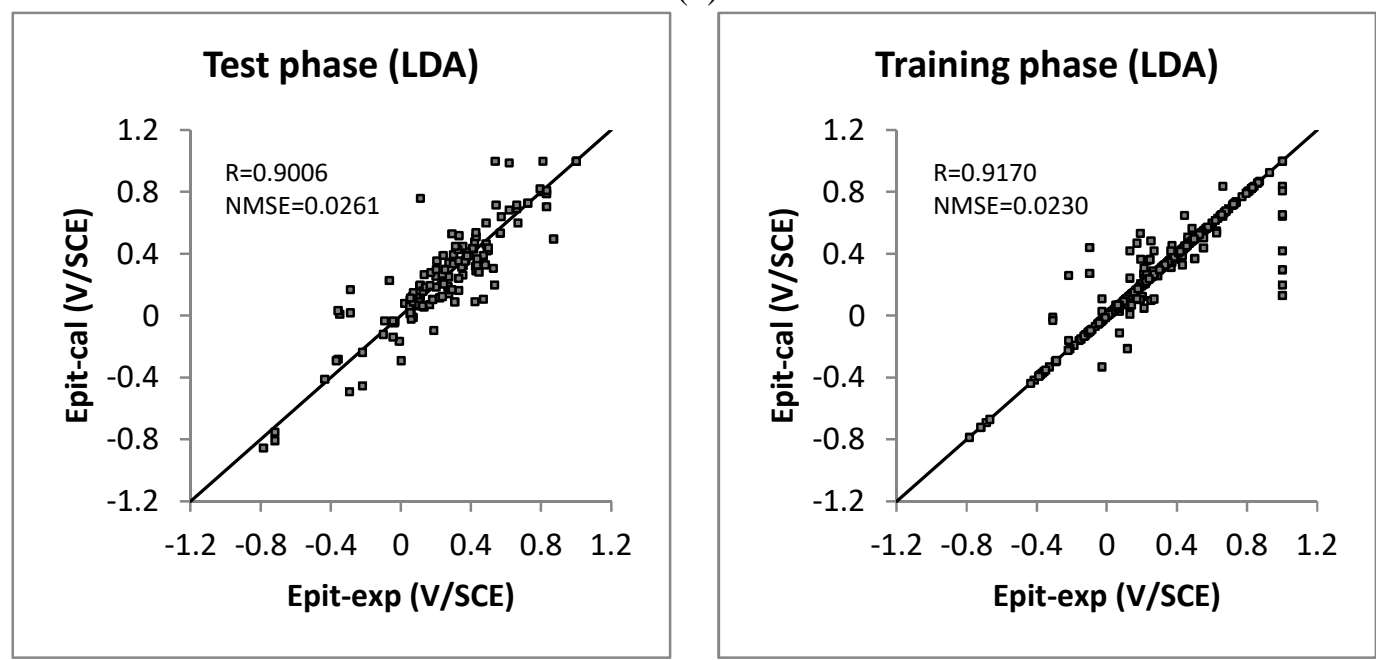

(i)

Fig.2. Calculated Epit versus experimental Epit during training and testing: (a) GA-ANN; (b) LSSVM; (c) PSO-ANN; (d) ANN; (e) ANFIS; (f) KNN; (g) KBP; (h) RT; (i) LDA

At first glance, we can see that all algorithms present acceptable prevision capabilities except LDA whose correlation coefficient remain below 0,91 during the test phase. In addition, KNN shows the best training performance with almost a perfect correlation coefficient.

In the electrochemical experiments, the scanning potential is limited to $1 \mathrm{~V} / \mathrm{SCE}$. Because beyond this limit starts the oxygen evolution which results in a significant increase in the current density that hides the current due to pitting phenomena. Through the distribution of the points we can see that some algorithms are unable to predict this limit. This situation can be seen through a vertical 
distribution of the points corresponding to $\mathrm{E}_{\text {pit-exp }}=1 \mathrm{~V} / \mathrm{SCE}$. KNN and ANN are more efficient in predicting a no-pitting situation.

\subsection{Comparison between algorithms:}

The need to compare all the algorithms, and at the same time to estimate for each algorithm the predictive capacity during training and testing phases led us to draw the following figure. In Figure 4, we can visualize for each algorithm the relationship between the correlation coefficient obtained during the test phase versus that obtained during the training phase.

Besides the obvious weak performance of LDA, the performance of other algorithms is relatively comparable. We can also see that a better training does not necessarily lead to a better performance during the test. KNN has always presented the best correlation coefficient during training as it is illustrated on figure 3 where all the points are located on the identity line. However for testing the algorithm is not the best one.

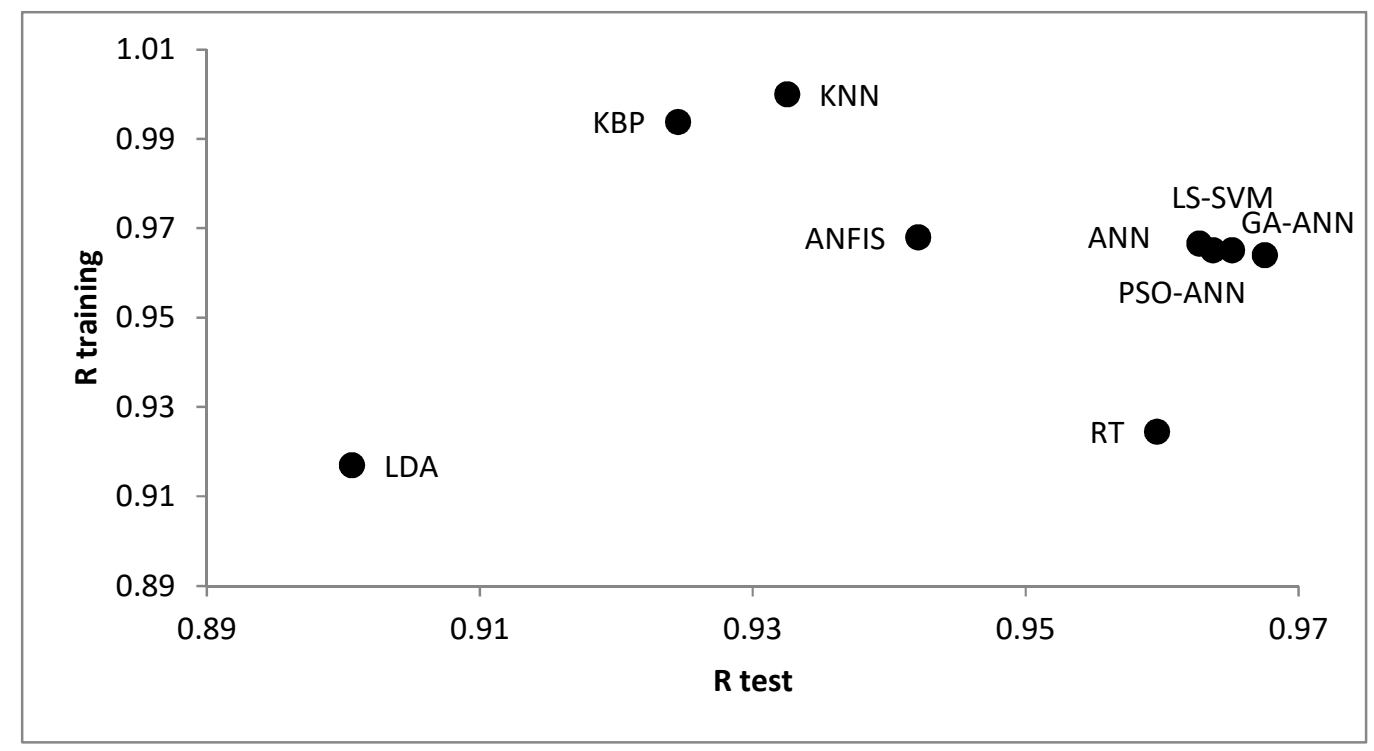

Fig.3. a 2D graphic indicating the performance order of algorithms for training and testing phases.

\subsection{Formatting of input data}

We also realized that the way by which the input data are presented to the algorithms allow a more or less successful pitting potential prediction. Figure 5 illustrates through histograms correlation coefficients $\mathrm{R}$ obtained with each algorithm during each phase when the concentrations of the reactants are presented in normal, square root or logarithmic form. 


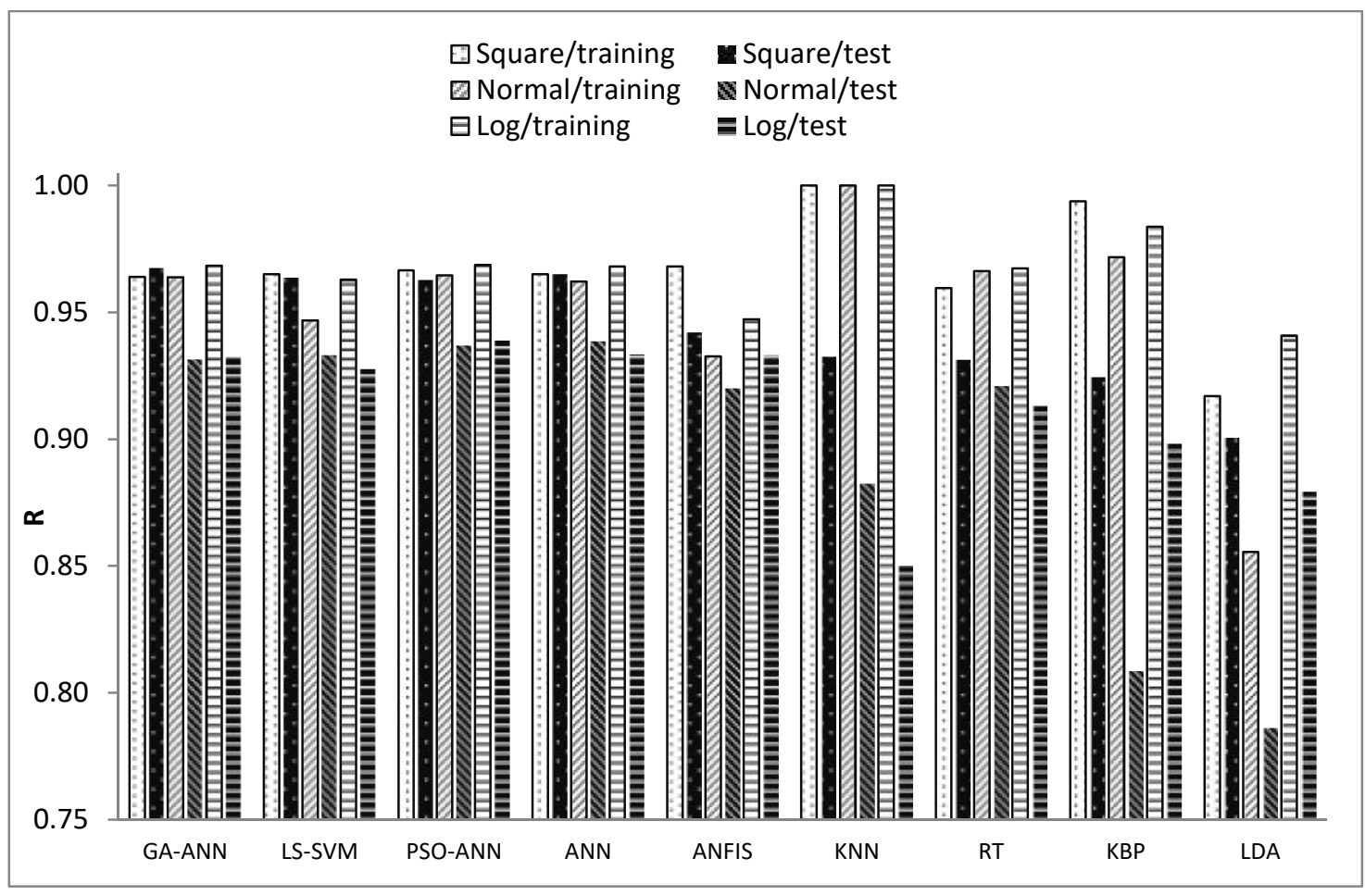

Fig.4. Histograms compiling correlation coefficients for all the algorithms, during training and testing and whether the concentrations of the reactants are taken in square root, normal or logarithm.

The histograms allow to clearly confirm the poor performance of LDA algorithm we mentioned above. We can also state that if KNN trains well, whatever the manner in which the chemical concentrations are presented, its generalization capabilities remain modest. Now regarding the way by which concentrations are presented, and except for LDA, all algorithms give a better correlation coefficient by considering the square root of the chemical concentrations.

\section{CONCLUSION}

Pitting corrosion and its inhibition remain microscopic phenomena that cannot be measured only by macroscopic means and observables such as the pitting potential. The difficulty to rigorously getting the pitting potential value is also enhanced by a probabilistic distribution around a mean value and that distribution is influenced by many parameters.

The evaluation of the performance of the algorithms considered in this study rejects clearly the LDA algorithm for this kind of phenomena. Although KNN remains the best algorithm during 
the training phase, in the testing phase the order of performance is: GA-ANN > LS-SVM > PSOANN $>$ ANN $>$ ANFIS $>$ KNN $>$ RT $>$ KBP $>$ LDA.

The manner by which the input data is presented to an algorithm has visibly an influence on the prediction performance. Considering the square root of the concentrations generally leads to a better correlation coefficient.

\section{ACKNOWLEDGEMENTS}

The authors are grateful to the Oum El Bouaghi University Algeria and Nuclear Research Center of Birine Algeria, for supporting the research work. In addition, the first author expresses special thanks to M. N. Boucherit for his great efforts.

\section{REFERENCES}

1. G.T. Burstein. Passivity and Localized Corrosion. Shreir's Corrosion 2010; 2:731-752, doi:10.1016/B978-044452787-5.00198-0

2. T. Suter, H. Bohni. A new microelectrochemical method to study pit initiation on stainless steels. Electrochimica Acta 1997;42(20-22):3275-3280, doi:10.1016/S00134686(70)01783-8

3. P. Ernst, R.C. Newman, Pit growth studies in stainless steel foils. I. Introduction and pit growth kinetics. Corros. Sci 2002;44(5):927-941, doi:10.1016/S0010-938X(01)00133-0

4. M. N. Boucherit, S. Amzert, F. Arbaoui, A. Sari, D. Tebib. Study of the evolution of a semi-open cooling circuit. Anti-corrosion methods and materials 2006;53(4):212-217, doi:10.1108/00035590610678901

5. M. N. Boucherit, S. Amzert, F. Arbaoui, S. Hannini, A. Hammache. Pitting corrosion in presence of inhibitors and oxidants. Anti-corrosion methods and materials 2008;55(3):155-122, doi : 10.1108/00035590810870419

6. M. Moreno, W. Morris, M.G. Alvarez, G.S. Duffo. Corrosion of reinforcing steel in simulated concrete pore solutions: Effect of carbonation and chloride content. Corros. Sci 2004;46(11):2681-2699, doi:10.1016/j.corsci.2004.03.013

7. O. Lopez-Garrity, G.S. Frankel. Corrosion Inhibition of AA2024-T3 By Sodium Silicate. Electrochimica Acta 2014;130:9-21, doi:10.1016/j.electacta.2014.02.117 
8. J.A.K Suykens; J. Vandewalle. Least squares support vector machine classifiers. Neural Processing Letters 1999;9(3):293-300, doi : 10.1023/A:1018628609742

9. Jian Jia, Chunshun Zhanga, Jayantha Kodikaraa, Sheng-Qi Yangb. Prediction of stress concentration factor of corrosion pits on buried pipes by least squares support vector machine. Engineering Failure Analysis 2015;55:131-138, doi:10.1016/j.engfailanal.2015.05.010

10. M.J. Jiménez-Comea, I.J. Turiasb, F.J. Trujillob. An automatic pitting corrosion detection approach for 316L stainless steel. Materials \& Design 2014;56:642-648, doi:10.1016/j.matdes.2013.11.045, doi:10.1016/j.corsci.2004.03.013

11. N. Morizet, N. Godin, J. Tang, E. Maillet, M. Fregonese, B. Normand. Classification of acoustic emission signals using wavelets and Random Forests : Application to localized corrosion. Mechanical Systems and Signal Processing 2016;70-71:1026-1037, doi:10.1016/j.ymssp.2015.09.025

12. R.F. de la Mare, Y.L. Bakouros, G. Tagaras. Understanding pipeline failures using discriminant analysis: the North Sea application. Reliability Engineering \& System Safety 1993;39(1):71-80, doi:10.1016/0951-8320(93)90149-S

13. J. Kennedy, R. Eberhart. Particle Swarm Optimization. Proceedings of IEEE International Conference on Neural Networks 1995;4:1942-1948, DOI: 10.1109/ICNN.1995.488968

14. Craig W. Reynolds. Flocks, herds and schools: A distributed behavioral model. ACM SIGGRAPH Computer Graphics 1987;21(4):25-34, doi: $10.1145 / 37402.37406$

15. A . Ismail, D.-S. Jeng, L.L. Zhang. An optimised product-unit neural network with a novel PSO-BP hybrid training algorithm: Applications to loaddeformation analysis of axially loaded piles. Engineering Applications of Artificial Intelligence 2013;26(10):2305-2314, doi:10.1016/j.engappai.2013.04.007

16. S.M. Mousavifard, M.M. Attar, A. Ghanbari, M. Dadgar. Application of artificial neural network and adaptive neuro-fuzzy inference system to investigate corrosion rate of zirconium-based nano-ceramic layer on galvanized steel in $3.5 \% \mathrm{NaCl}$ solution. Journal of Alloys and Compounds 2015;639:315-324, doi:10.1016/j.jallcom.2015.03.052 
17. J. H. Holland. Adaptation in Natural and Artificial Systems. Univ. of Michigan Press, Ann Arbor, MI, 1975.

18. R. Machavaram, C. J. Prakash, R. Hifjur. Prediction of optimized pretreatment process parameters for biodiesel production using ANN and GA. Fuel 2009;88(5):868-875, doi:10.1016/j.fuel.2008.12.008

19. Fatih Altun, Tamer Dirikgil. The prediction of prismatic beam behaviours with polypropylene fiber addition under high temperature effect through ANN, ANFIS and fuzzy genetic models. Composites Part B: Engineering 2013;52:362-371, doi:10.1016/j.compositesb.2013.04.015

20. L. Breiman, J.H. Friedman, R.A. Olshen, C.J. Stone, Classification and Regression Trees, First Ed. Wadsworth, Belmont, USA, 1984.

21. V. Guigue, A. Rakotomamonjy, S. Canu. Kernel Basis Pursuit. Revue d'Intelligence Artificielle 2006;20(6):757-774, doi:10.3166/ria.20.757-774

22. Yafei Wang, Guangxu Cheng, Wei Wu, Qiao Qiao, Yun Li, Xiufeng Li. Effect of pH and chloride on the micro-mechanism of pitting corrosion for high strength pipeline steel in aerated $\mathrm{NaCl}$ solutions. Applied Surface Science. 2015,349: 746-756, doi:10.1016/j.apsusc.2015.05.053

23. H.E.H. Bird, B.R. Pearson, P.A. Brook. The breakdown of passive films on iron. Corros. Sci 1988;28(1):81-86, doi:10.1016/0010-938X(88)90009-1

24. P. Marcus, V. Maurice, H.H. Strehblow. Localized corrosion (pitting): a model of passivity breakdown including the role of the oxide layer nanostructure. Corrosion Science 2008;50(9):2698-2704, doi:10.1016/j.corsci.2008.06.047.

\section{How to cite this article:}

Boukhari Y, Boucherit M. N, Zaabat M, Amzert S, Brahimi K. Artificial intelligence to predict inhibition performance of pitting corrosion. J. Fundam. Appl. Sci., 2017, 9(1), 308-322. 